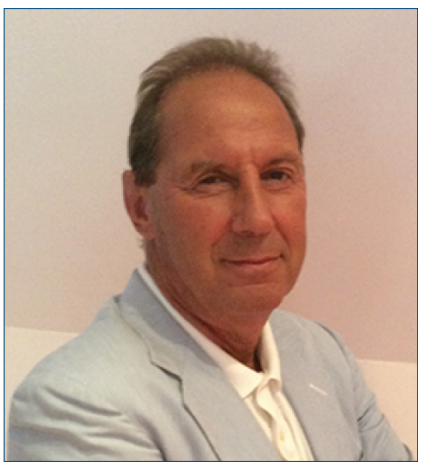

IAIN HUTCHISON, BDS, MBBS, FRCS Edin, FRCS Eng, FFDRCSI, FDSRCS

lain Hutchison, BDS, MBBS, FRCS Edin, FRCS Eng, FFDRCSI, FDSRCS, treats patients with diseases, deformity, and injuries affecting the most socially important part of our bodies - the face.

Professor Hutchison created the Saving Faces Art Project, which charts the physical and emotional journey of facia surgery patients. The Saving Faces Art Project has had a dramatic effect on the public and proved cathartic for the patients who participated. The paintings have toured the world and have been seen by $>2$ million people.

He strives to demystify medicine and has participated in many TV and radio programmes about facial surgery. Several of his lectures are available for view on the internet including a TED talk entitled "Saving Faces."

He wants to improve patient treatment through clinical research. In 2000, he founded Saving Faces (www.savingfaces.co.uk), which leads international multicenter clinical trials on facial disease and injury prevention and treatment. In October 2019, Saving Faces published the first nationwide mouth cancer surgical research, the SEND study, which will save thousands of lives every year.

He also founded the world's only National Facial, Oral, and Oculoplastic Research Centre (www.nforc.co.uk). Through its research this center will revolutionise treatment for patients with facial diseases, deformity, and injury worldwide. He also encourages surgeons to introduce clinical research into their day-to-day practice.

He wants to inspire and train the doctors of the future and gives frequent careers talks in schools and universities and is in great demand as a scientific and public lecturer.

doi: 10.6004/jnccn.2021.7036

The ideas and viewpoints expressed in this commentary are those of the author and do not necessarily represent any policy, position, or program of NCCN.

\section{More Evidence From a US Nationwide Cohort Study for the Survival Benefit of END in Early Mouth Cancer: Time for a Change in Guidelines}

\author{
lain Hutchison, BDS, MBBS, FRCS Edin, FRCS Eng, FFDRCSI, FDSRCS
}

$\mathbf{M}$ outh cancer, or oral squamous cell carcinoma (OSCC), is the 11th most common malignancy. It can be the most socially disabling cancer, affecting appearance, speech, and eating, yet clinical research to improve treatment is very poorly funded.

Therefore, the research by Wushou et al ${ }^{1}$ on early mouth cancer, reported elsewhere in this issue, in combination with reports from 2 rare prospective randomised research studies-one from Tata Memorial Centre in Mumbai in $2015^{2}$ and a nationwide study from Britain with a meta-analysis in 2019 (the SEND trial) ${ }^{3}$-merit an opportunity to modify guidelines regarding the treatment of early mouth cancer.

The article by Wushou et $\mathrm{al}^{1}$ concentrates on clinical stage T1NOMO (cT1NOMO) OSCC. They identified and analyzed data on 5,752 patients from the $\mathrm{NCI}$ SEER database, comparing overall survival (OS) and disease-specific survival (DSS) in patients who underwent tumor resection with or without elective neck dissection (END). They found only one previous prospective randomised trial (SEND) that specifically quantified the benefit of END in cT1NO OSCC. ${ }^{3}$ The paper on this trial showed that END was beneficial for small tumors (pT1 stage) or tumor diameter $\leq 10 \mathrm{~mm}$, with corresponding disease-free survival (DFS) hazard ratios (HRs) of 0.49 and 0.63 , respectively. ${ }^{3}$ In fact, the Tata Memorial trial also reported an OS benefit for END with T1NO tumors, with HRs of approximately 0.75. ${ }^{2}$ Therefore, the British paper concluded that together, it and the Tata Memorial study provided evidence of the benefit for END regardless of tumor size. ${ }^{3}$

Zhan et $a^{4}$ in 2018 reported on patients with cT1NO OSCC who were treated with surgical resection and END. Their data was abstracted from the National Cancer Database. They identified 2,623 (15\%) patients with occult neck disease and studied the relationship between tumor differentiation and the presence of occult neck disease. They concluded that END should be recommended for all moderately ( $17.4 \%$ occult neck disease) and poorly differentiated (28.5\% occult neck disease) cT1NO OSCC, regardless of the depth of invasion. They suggested that clinicians could consider not performing END in well-differentiated tumors, for which they found only $5.9 \%$ of patients had occult neck disease. ${ }^{4}$ Yet in the SEND trial, the DFS HR for this subgroup of patients with well-differentiated OSCC was 0.54, suggesting benefit for END even in those with pT1 stage tumors that are well-differentiated. ${ }^{3}$

This discrepancy between pathologic findings of only $5.9 \%$ of occult disease but clinical benefit for END suggested by the SEND trial could be explained by the presence of micrometastases and isolated tumor cells that are not identified on routine pathologic examination. ${ }^{5}$ The detection of these, which are reported to be present in $8.8 \%$ of pathologically node-negative necks, would upstage disease in these patients. It suggests that standard pathologic examination may underestimate the percentage of patients with

See page 385 for related article. 
occult neck disease that, if left untreated, will manifest later and require therapeutic neck dissection. And it lends further support to the concept of offering END in early mouth cancer, as the surgery clears this previously undetected "super" occult neck disease. ${ }^{5}$

Wushou et al" "aimed to gather sufficient evidence to make strong well-supported recommendations to either support or refute the findings of [this] single published study," specifically evaluating whether performing END at the same time as local tumor resection confers benefit for OS and DSS in patients with cT1NOMO OSCC. They also studied certain prognostic factors, such as age, sex, marital status, tumor differentiation, and tumor site.

Of incidental interest was their finding that being married had a significantly protective effect, associated with improved OS and DSS. They did not report whether unmarried women fared better, worse, or the same as unmarried men. This finding regarding the association of marital status and increased survival was also reported in another study in 2017 using SEER data from 11,022 patients. ${ }^{6}$ The investigators found that married patients were more likely to be diagnosed at an earlier stage, and postulated that family support was valuable. ${ }^{6}$

Wushou et al $^{1}$ reported some limitations with their study. In the British meta-analysis, the findings of the Mumbai $^{2}$ and British studies ${ }^{3}$ complemented each other, generating even more valuable information. As Wushou et al point out, their study now complements the Mumbai and British studies. Similarly, the findings from these 2 studies can address and resolve Wushou et al's limitations.

First, they did not report depth of invasion. ${ }^{1}$ The SEND paper conducted analyses according to the 8th edition of the AJCC Cancer Staging Manual.' The perprotocol OS HRs were 0.76 for T1, 0.64 for T2, and 0.73 for T3, respectively, with corresponding DFS HRs of 0.51 , 0.75 , and 0.75 , confirming that even patients with favourable staging still benefit from END. ${ }^{3}$

Second, Wushou et $\mathrm{al}^{1}$ are concerned that research data bias may potentially exist due to surgeon bias and patient choice. The British paper recognised this and gained ethical approval at an early stage to include eligible patients who were not approached or declined randomization but agreed to follow up. They were analysed separately to the randomised group as a form of realworld cohort. Their results mirrored the results of the main trial and are reported in the paper. ${ }^{3}$

Finally, Wushou et al ${ }^{1}$ were concerned that they did not evaluate the long-term complications of neck dissection or lymph node metastasis after surgery. The British paper followed patients for 5 years, reporting on survival, recurrences, and new tumors, as well as complications, adverse effects, quality-of-life outcomes, and resource use, and therefore captured all these events. ${ }^{3}$ In that study, the investigators reported that more patients experienced an adverse effect of any grade in the END group, but there was no significant difference in serious events. Although neck sensory and motor nerve effects and swallowing problems were more common in patients who underwent $E N D$, the procedure had minimal impact on most qualityof-life components. ${ }^{3}$ END increased the length of hospital stay from a median of 2 days for tumor resection only to 6 days for END. Up to 2 years postoperatively the resource use was the same for END.

Wushou et al" state that their "findings showed that END significantly improved both OS and DSS in these patients, which is consistent with the findings of 2 largescale prospective randomized studies and a meta-analysis of all prospective randomized studies. Our retrospective analysis using the SEER database to analyze 5,752 patients with only CT1NOMO OSCC complements those studies, and together with the meta-analysis provides evidence supporting a review of head and neck cancer guidelines."

The President of The Union for International Cancer Control submitted a comment on the paper by Hutchison et $\mathrm{al}^{3}$ to the British Journal of Cancer, stating that "despite the highest form of evidence by way of 2 large randomised trials in favour of an [END]... We stand the risk of subjecting our patients to poorer disease control by observing the neck in spite of having irrefutable evidence in favour of END. ${ }^{18}$

In light of these 2 comments, ${ }^{1,8}$ I reviewed the current NCCN Clinical Practice Guidelines in Oncology (NCCN Guidelines) for Head and Neck Cancers (Version 1.2021). ${ }^{9}$ In the Principles of Surgery [SURG-A, available at NCCN.org] section, there are 11 references dating from 1978 to 2016, including 7 references on sentinel lymph node (SLN) biopsy. The guidelines state that "SLN biopsy is an alternative to [END] for identifying an occult cervical metastasis in patients with early (T1 or T2) oral cavity carcinoma in centers where expertise for this procedure is available."

A Cochrane review from $2018^{10}$ states, "The evidence is insufficient to draw conclusions about [END] of clinically negative neck nodes." This 2018 review precedes the 2019 SEND trial, ${ }^{3}$ which resolves a second issue raised in the Cochrane review: "Reporting of adverse events in all trials was poor and it was not possible to compare the quality of life...." 10

Although this Cochrane systematic review ${ }^{10}$ was the most recent reference cited in the "Cancer of the Oral Cavity" section of the NCCN Guidelines, there are now 3 papers, 2 of which were published after 2018, showing OS, DFS, and DSS benefit for END in patients with CT1NO and CT2NO OSCC: a prospective randomised study (PRS) from a renowned Indian tertiary centre ; a European nationwide PRS with 5-year follow-up, realworld cohort, and meta-analysis ${ }^{2}$; and now big data from a renowned database of American patients. ${ }^{1}$ 
In the face of this new, powerful evidence from diverse sources across 3 continents, all ethics committees would probably regard it as unethical to conduct another PRS on this topic, so it is unlikely that any further, new, high-level research evidence will be produced. Therefore, isn't it time to modify the guidelines and provide unequivocal support for the survival benefit of END in early mouth cancer?

There is now quantifiable information on the benefits for survival with END and also the complications and resource implications of selecting either END or local resection only. This knowledge enables surgeons to inform patients and their families about the benefits and drawbacks of opting for or against END, and thereby allows them to participate in treatment decision-making. Now that the age of the paternalistic doctor is over, the guidelines should also recommend that doctors offering END to all NO patients should do so explaining all this quantifiable evidence about relative benefits for their disease stage and the percentage risk of complications. Armed with good evidence, we can help our patients make the best choice for themselves and their family.

Disclosures: The author has disclosed no financial interests, arrangements, or affiliations with the manufacturers of any products discussed in this article or their competitors.

Correspondence: lain Hutchison, BDS, MBBS, 2 Eton Road, London NW3 4SP, United Kingdom. Email: hutch.london@googlemail.com

\section{References}

1. Wushou A, Wang M, Yibulayin F, et al. Patients with cT1NOMO oral squamous cell carcinoma benefit from elective neck dissection: a SEER-based study. J Natl Compr Canc Netw 2021;19:385-392.

2. D'Cruz AK, Vaish $R$, Kapre $N$, et al. Elective versus therapeutic neck dissection in node-negative oral cancer. N Engl J Med 2015;373:521-529.

3. Hutchison IL, Ridout F, Cheung SMY, et al. Nationwide randomised trial evaluating elective neck dissection for early stage oral cancer (SEND study) with meta-analysis and concurrent real-world cohort. Br J Cancer 2019;121:827-836.

4. Zhan KY, Morgan PF, Neskey DM, et al. Preoperative predictors of occult nodal disease in CT1NO oral cavity squamous cell carcinoma: review of 2623 cases. Head Neck 2018:40:1967-1976.

5. Majumdar KS, Rao VUS, Prasad R, et al. Incidence of micrometastasis and isolated tumour cells in clinicopathologically node-negative head and neck squamous cell carcinoma. J Maxillofac Oral Surg 2020;19:131-135.
6. Shi X, Zhang TT, Hu WP, et al. Marital status and survival of patients with oral cavity squamous cell carcinoma: a population-based study. Oncotarget 2017;8:28526-28543.

7. Amin MB, Edge S, Greene F, et al, eds. AJCC Cancer Staging Manual, 8th ed. Basel, Switzerland: Springer International Publishing; 2017.

8. Dhar H, Vaish R, D'Cruz AK. Comment on "Nationwide randomised trial evaluating elective neck dissection for early stage oral cancer (SEND study) with meta-analysis and concurrent real-world cohort." Br J Cancer 2020;123:1198-1199.

9. Pfister DG, Spencer S, Adelstein D, et al. NCCN Clinical Practice Guidelines in Oncology: Head and Neck Cancers. Version 1.2021. Accessed March 1, 2021. To view the most recent version, visit NCCN.org

10. Bulsara VM, Worthington HV, Glenny AM, et al. Interventions for the treatment of oral and oropharyngeal cancers: surgical treatment. Cochrane Database Syst Rev 2018;12:CD006205. 